\title{
Flying into the Sun
}

\author{
After 60 years of technological and materials development, in August this year the Parker Solar Probe set off on its \\ journey to skim the atmosphere of the Sun. Mission Scientist Adam Szabo summarizes this ambitious adventure.
}

T he upper solar atmosphere - the corona - is one of the last completely unexplored regions of the heliosphere. The NASA Parker Solar Probe, named after Eugene Parker and launched on 12 August 2018, will chart this region during its seven-year nominal mission during which it will spend 440 hours inside 15 solar radii. At its closest, it will descend to $\sim 10$ solar radii $\left(R_{\odot}\right)$, seven times closer to the Sun than any previous spacecraft (Fig. 1).

The primary science objectives of the mission are threefold: to reveal why the solar corona is significantly hotter than the regions lying below it, how the solar wind particles are accelerated to supersonic and super-Alfvénic speeds, and what physical processes are responsible for the creation of solar energetic particles (SEPs).

These fundamental questions date back to the 1958 National Research Council Space Study Board recommendations, but a mission to address these questions had to wait until required technologies primarily high-temperature materials could be developed.

The solar corona can reach a temperature of several million kelvin, much higher than the underlying atmospheric layers. Several theories have been developed to explain this behaviour, from the release of magnetic energy by continuous small solar eruptions ('nanoflares'), to the transport of energy through the atmosphere by different kinds of waves, to turbulent fluctuations heating protons and ions. The Parker Solar Probe will provide in situ electromagnetic and plasma measurements to distinguish between these theories.

The solar wind emanating from coronal holes located at high solar latitudes has higher speeds than the near-equatorial slow solar wind. However, there is considerable uncertainty where this slow solar wind originates from, because at Earth's distance the individual blobs of solar wind have merged into a more homogeneous flow. The Parker Solar Probe will be able to observe the intermittent nature of the solar wind near its origin.

Finally, the Parker Solar Probe will help determine the origins of SEPs, solar particles travelling nearly at the speed of light and responsible for disrupting space-borne

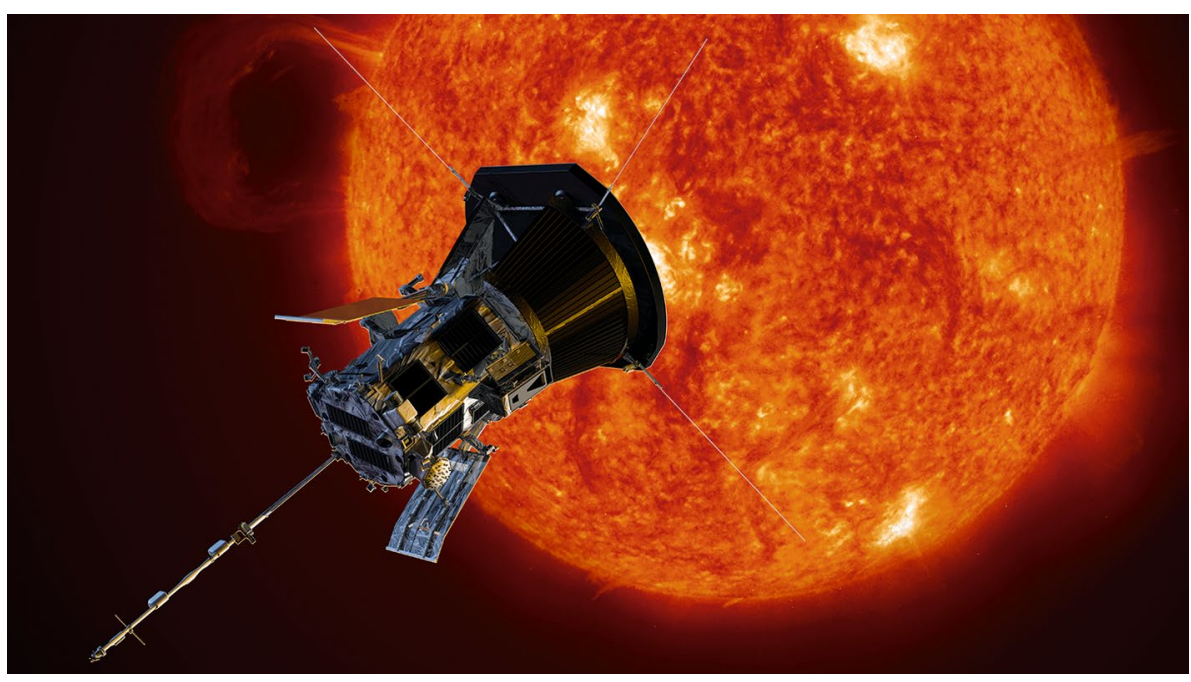

Fig. 1 | An artist's representation of the Parker Solar Probe. FIELDS suite antennae and the magnetometer instruments are visible. Credit: NASA/Johns Hopkins APL/Steve Gribben

electronics. They are associated with solar flares and coronal mass ejection-driven interplanetary shocks. But the detailed mechanism of particle acceleration is unknown. None of the current theories (for instance, magnetic reconnection or shock acceleration) can produce $10-100 \mathrm{MeV}$ SEPs from the pool of the thermal solar wind. The Parker Solar Probe will measure for the first time the source populations and the details of the electromagnetic fields accelerating the particles in the very region where SEPs are generated.

The Parker Solar Probe carries instruments measuring electric and magnetic fields and charged particles from low thermal solar wind energies to those of SEPs. The FIELDS suite includes electric and magnetic field instruments that measure both types of fields from near constant values to very high frequencies. Such a range is necessary to capture the forces that accelerate and heat the solar charged particles. The SWEAP suite is composed of particle detectors sensitive to thermal solar wind particles. The SWEAP instruments will measure the particle distribution functions at an unprecedented sub-second time cadence that is necessary to identify modes of waveparticle interactions. The IS $\odot I S$ instruments extend the Parker Solar Probe energy range to include accelerated SEPs. Finally, two side-looking cameras, WISPR, will provide contextual images. An innovative carboncarbon composite heat shield will protect the instruments from the extreme heat of the Sun, reducing temperatures of $1,000-1,500 \mathrm{~K}$ to $300 \mathrm{~K}$, and retractable water-cooled solar panels will provide the requisite energy. These new technologies became available only recently, making this mission finally, after 60 years, possible.

The launch of the Parker Solar Probe is a culmination of eight years of hard work by a large team of engineers and scientists at the Johns Hopkins University Applied Physics Laboratory, NASA Centers, the University of California, Berkeley, Smithsonian Astrophysical Observatory, Southwest Research Institute, the Naval Research Laboratory, and the University of Michigan.

Adam Szabo

NASA Goddard Space Flight Center, Greenbelt, MD, USA.

e-mail: Adam.Szabo@nasa.gov

Published online: 17 September 2018 https://doi.org/10.1038/s41550-018-0580-3 